\title{
Should every survivor of a heart attack be given a beta-blocker?
}

\author{
Part I Evidence from clinical trials
}

\author{
J R HAMPTON
}

In 1965 Snow published a study which appeared to show that the fatality rate of patients who had had a heart attack was reduced if they were treated with propranolol. ${ }^{1}$ Unfortunately, this study was neither properly randomised nor double-blind, but its results led to an interest in the use of beta-blockers in patients who have had a heart attack that has lasted nearly 20 years. Since 1965 numerous studies have been published, but until a year ago no clear pattern had emerged in their results. Most of these studies were too small to show anything other than an enormous effect, and many had other faults of design or execution. The only large trial published before 1981 was that of practolol, ${ }^{2}$ and this drug had to be withdrawn because of its side effects. Now, however, four more large trials have been completed and at last we are in a position to consider whether the time has arrived for a wide scale change in clinical practice, with all survivors of heart attacks henceforth being treated with a beta-blocker. Perhaps one-third of patients who have had a heart attack will have high blood pressure or angina and so will have a conventional indication for beta-blockade; in the first part of this report and the two following parts we shall be concerned with the prophylactic use of beta-blockers in patients without these problems.

\section{Early and late treatment}

The place for beta-blockers in the first few hours after the onset of the symptoms of a heart attack and in the period when the acute illness

Paper presented at the plenary session of the annual meeting of the British Cardiac Society, held at Nottingham, April 1982.

Department of Medicine, University Hospital, Queen's Medical Centre, Nottingham

J R HAMPTON, DPHIL, FRCP, professor of cardiology is Jver should be considered separately. It is difficult to separate treatment of one episode from prevention of a second, but in the initial phase the main emphasis of treatment has to be on the relief of symptoms and the correction of arrhythmias and heart failure; later the main aim is to prevent sudden death and reinfarction. The mechanism of death is not necessarily the same in the first few hours or days after a heart attack as it is later on, so a drug that is useful early might well be ineffective later. The precise mechanism by which beta-blockers might act is not known; they are not particularly powerful antiarrhythmic agents, ${ }^{3}$ and while they have complex effects on cellular biochemistry it is far from clear which of these are important. It is, however, certain that in the initial stages of a heart attack beta-blockers may be difficult to use, for patients then have an unstable heart rate and blood pressure, and if beta-blockers are given it can be difficult to know whether bradycardia and hypotension result from medication or from the underlying disease. There is no 9 certain evidence that beta-blockers cause heart failure in patients with heart attacks, ${ }^{45}$ but many clinicians fear that this may happen. In of acutely ill patients any benefit beta-blockers might confer in some patients would possibly be outweighed by harm done to others.

Early and late trials need fundamental differences in design. In an $N$ early trial the fatality rate in patients with heart attacks given placebo treatment can be expected to be high-perhaps $20 \%$ or more, depending on age and on the type of patient included in the trial. Thus, the number of patients needed for a significant difference between treated $\Theta$ and placebo groups to be found is relatively small. If entry to the trial is delayed for three or four weeks the expected fatality rate in the ? next year will he only between $5 \%$ and $10 \%$, so the trial will have to 0 be large. The design of late entry trials is simplified by the fact that only patients with confirmed infarctions need to be included; in early entry trials patients who are eventually found not to have had a heart attack are bound to be included, and this can make the interpretation of results difficult.

The division between "early" and "late" trials is entirely arbitrary, 8 but a cut-off point at 72 hours is convenient. If the only useful effect of beta-blockers is to limit necrosis of ischaemic myocardium then treatment begun later than four or five hours may be too late, but up to now no such "very early" trials have been reported. 


\section{EARLY ENTRY TRIALS}

The results of all the early entry trials that were properly randomised and double-blind, and which contained reasonable (if not adequate) numbers of patients, are shown in fig 1 . In this method of presenting data $^{6}$ the difference in fatality rate between treated and placebo groups is expressed as a percentage, so that if the fatality rate in the placebo group was $12 \%$ and in the treated group $8 \%$, the result would be described as a $30 \%$ reduction in mortality associated with active treatment. In some trials the treated patients fared worse than those given placebo, and for these the percentage increase in mortality is calculated. In fig 1 the horizontal bars represent the $95 \%$ confidence limits for the results of each trial; within each bar the vertical line represents the actual result. The maximum decrease in mortality associated with active treatment is $100 \%$, for this indicates no deaths; an increase in mortality of more than $100 \%$ is possible, so the bars are asymmetrically placed about the line of zero effect. The results of the early entry trials are scattered around the line of no effect: in some trials treatment with a beta-blocker was harmful, in some it seemed neutral, and in others it seemed beneficial (fig 1). The results of all the trials are compatible with benefit from active treatment (that is, the $95 \%$ confidence intervals extend to the right of the line of zero effect) but in only one trial, of metoprolol, ${ }^{7}$ were the results not also compatible with harm.

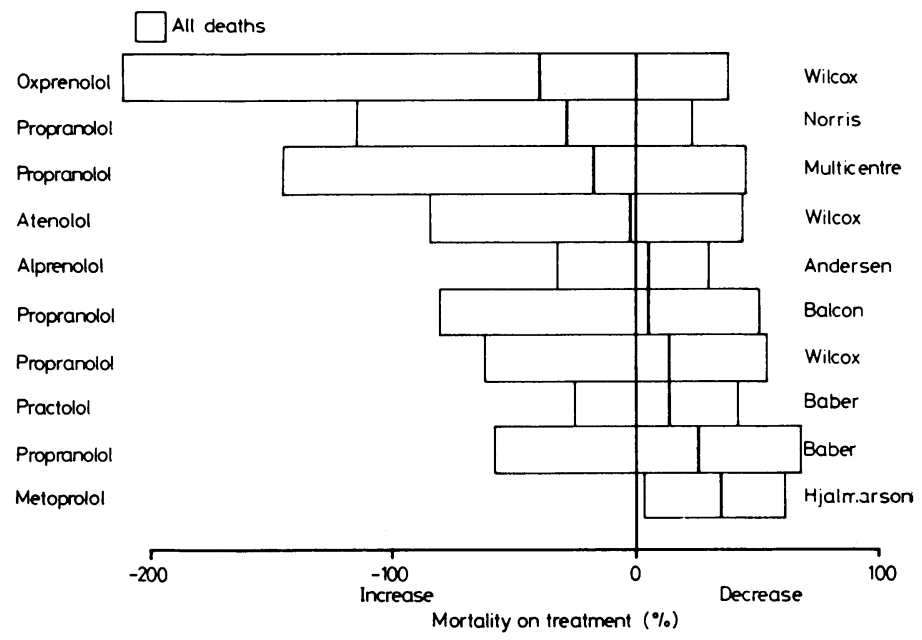

FIG 1-Results of postinfarction trials with beta-blockers in which treatment was begun within 72 hours of the onset of symptoms showing $95 \%$ confidence intervals (for explanation see text). ${ }^{6}$

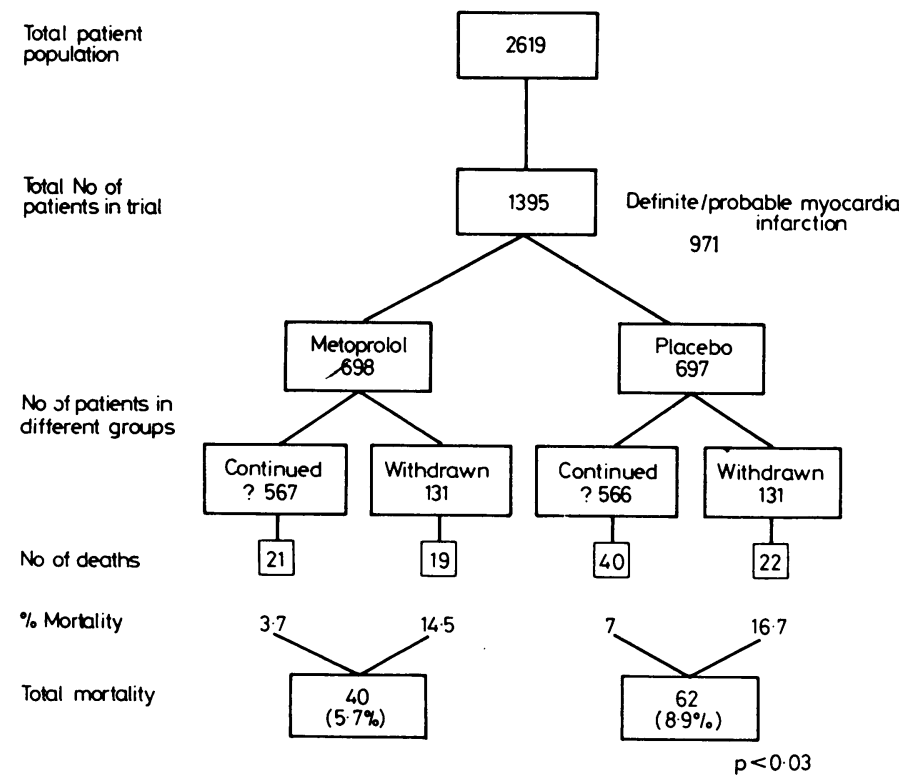

FIG 2-Comparison of the effect of metoprolol (15 mg intravenously, $200 \mathrm{mg}$ daily) and placebo on the mortality of patients with myocardial infarction.?

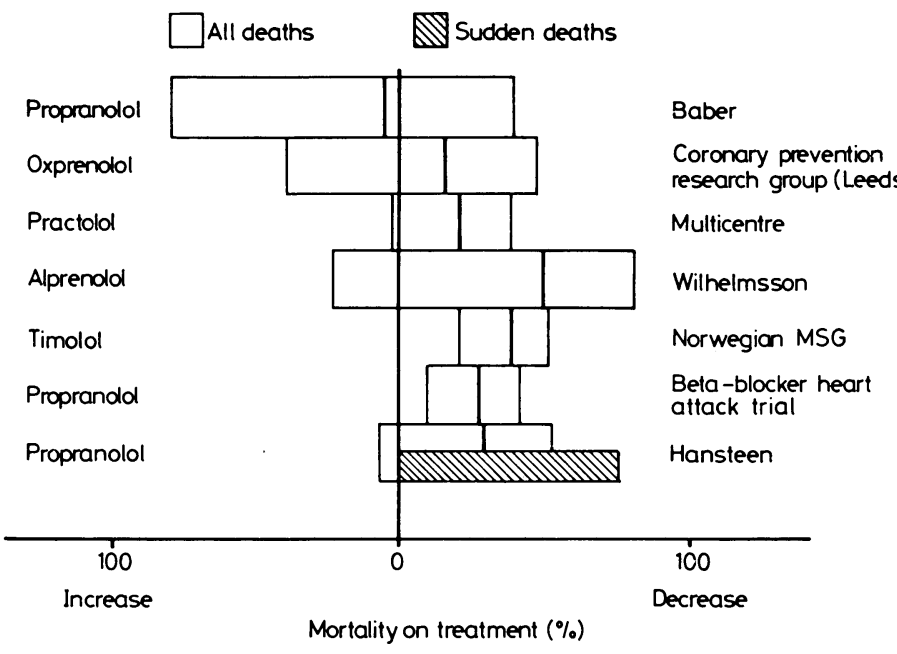

FIG 3-Results of trials in which treatment with a beta-blocker was begun more than 72 hours after the onset of symptoms showing $95 \%$ confidence intervals. $^{6}$

In fig 2 the results of the metoprolol trial are presented in a standard format that allows trials to be compared easily. ${ }^{8}$ Rather more than half the potential patients were included in the study, so the results should be generally applicable. As is inevitable in early entry trials, there was a relatively high proportion of patients who were eventually found not to have had an infarction (424 of 1395, 31\%) and this contributed to the low fatality rate for an early entry trial of $8.9 \%$ in the placebo group. The withdrawal rate in both groups was 131 of $697(19 \%)$ but whether analysed on an explicative or pragmatic (intention to treat) basis, there was a reduced mortality in the treated group; the difference on the "intention to treat" analysis just achieved conventional levels of statistical significance, $p$ being $<0.03$. This trial $^{7}$ is the largest and best designed of the early entry trials. Had the previous studies mainly suggested a benefit from active treatment it might have clinched the case for early treatment with a beta-blocker of all patients with suspected myocardial infarction. In view of the adverse results of some trials, however, and the undoubted problems associated with the use of beta-blockers in acutely ill patients, it is probably not yet justified to advise that all such patients should be given prophylactic treatment.

\section{LATE ENTRY TRIALS}

Fig 3 shows the $95 \%$ confidence limits for the late entry trials. ${ }^{6}$ In only one did the result favour placebo treatment and was statistically compatible with either benefit or harm from active treatment. In all the rest the results favoured beta-blockers, and this trend is made convincing by the fact that in two trials (the Norwegian timolol ${ }^{9}$ and the BHAT $^{5}$ propranolol studies) the results were compatible only with benefit (figs 4 and 5 ).

A comparison of these results shows that the timolol study included a higher proportion of potential patients and also comprised a higher risk population than the BHAT study. It is curious that patients given timolol had a lower mortality than those given placebo whether they continued to take the drug or not; the data so far provided in the BHAT study do not show what happened in this respect. The timolol trial showed the greatest benefit from active treatment, but the results of the BHAT study were numerically very similar to those of the practolol trial ${ }^{2}$ (fig 6). Treatment with practolol did not quite achieve a statistically significant difference from that with placebo. The practolol trial was only of academic interest becaus a the drug is no longer available, but its results can now be seen to add considerable weight to the case for using beta-blockers "late" after myocardial infarction.

In several of these trials treatment with a beta-blocker reduced sudden death as well as total mortality, but none of these studies was designed specifically to find out whether active treatment had a particular effect on sudden death. It is not acceptable simply to declare multiple end-points before a trial is begun, for the more the end-points the more likely it is that a statistically significant difference between treated and placebo groups will occur by chance. In one studyconducted in Norway and using propranolol ${ }^{10}$ - a reduction in sudden 
death was one of the end-points and the number of patients included in the trial was calculated on the basis of the predicted number of sudden deaths in the placebo group. Fig 7 shows the results of this trial, which was unusual in aiming to include only high-risk patients. In addition to low-risk patients, a relatively large number of very high-risk patients had to be excluded, and the overall fatality rate in the placebo group at the end of one year was not particularly high at $13 \%$ (fig 7). Active treatment appeared to reduce total mortality, although the difference between treated and placebo groups was not statistically significant; there was, however, a significant reduction in sudden death.

Although the reduction in sudden death in the Norwegian trial ( $8 \%$ in the placebo group and $4 \%$ in the group given propranolol) was less dramatic than that found in one of the early alprenolol trials ${ }^{11}$ $(9.5 \%$ in the placebo group and $0.9 \%$ in the treated group) there can now be little doubt that beta-blockers reduce the risk of sudden death in patients after infarction. Thus late treatment with a beta-blocker reduces mortality after a myocardial infarction, but it is obvious that many patients have to be treated for relatively few lives to be pro-

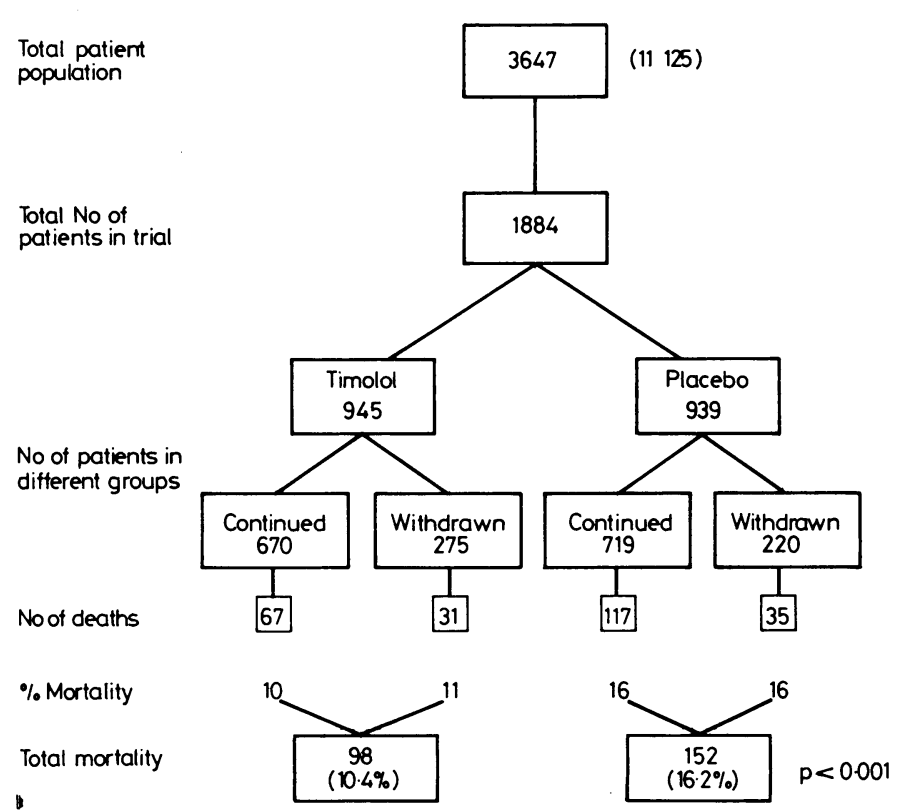

FIG 4-Comparison of the effect of timolol ( $10 \mathrm{~g}$ twice daily from 7-28 days to 17 months) and placebo on mortality of patients (aged 20-75 years) with myocardial infarction (Norwegian Multicentre Study). ${ }^{9}$

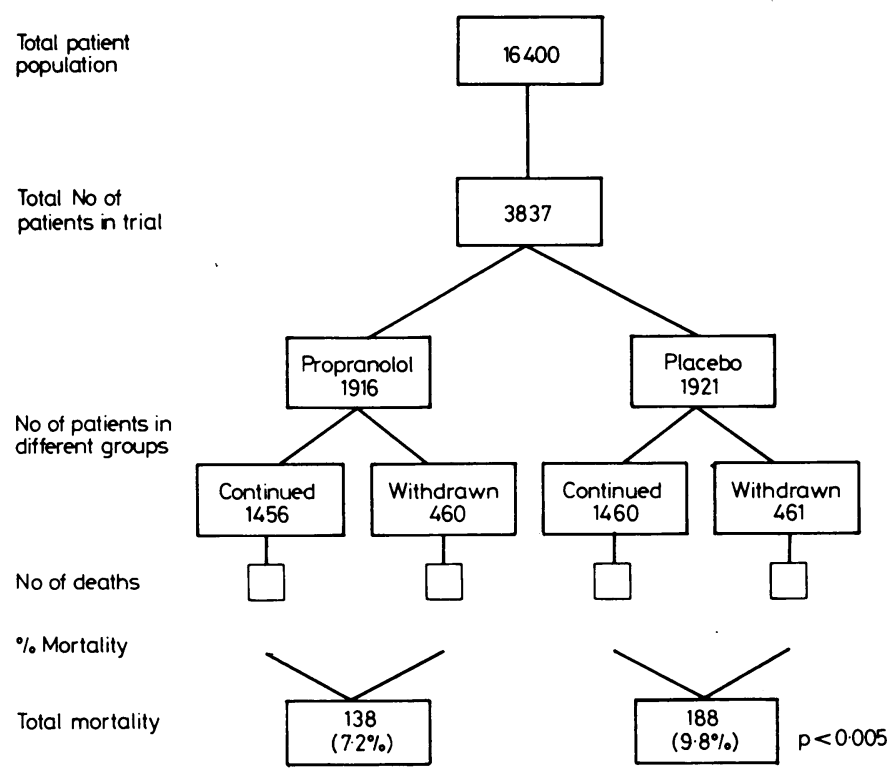

FIG 5-Comparison of the effect of propranolol (120-240 mg daily) and placebo on the mortality of patients (aged 30-90 years) 14 days after myocardial infarction (BHAT study). ${ }^{5}$

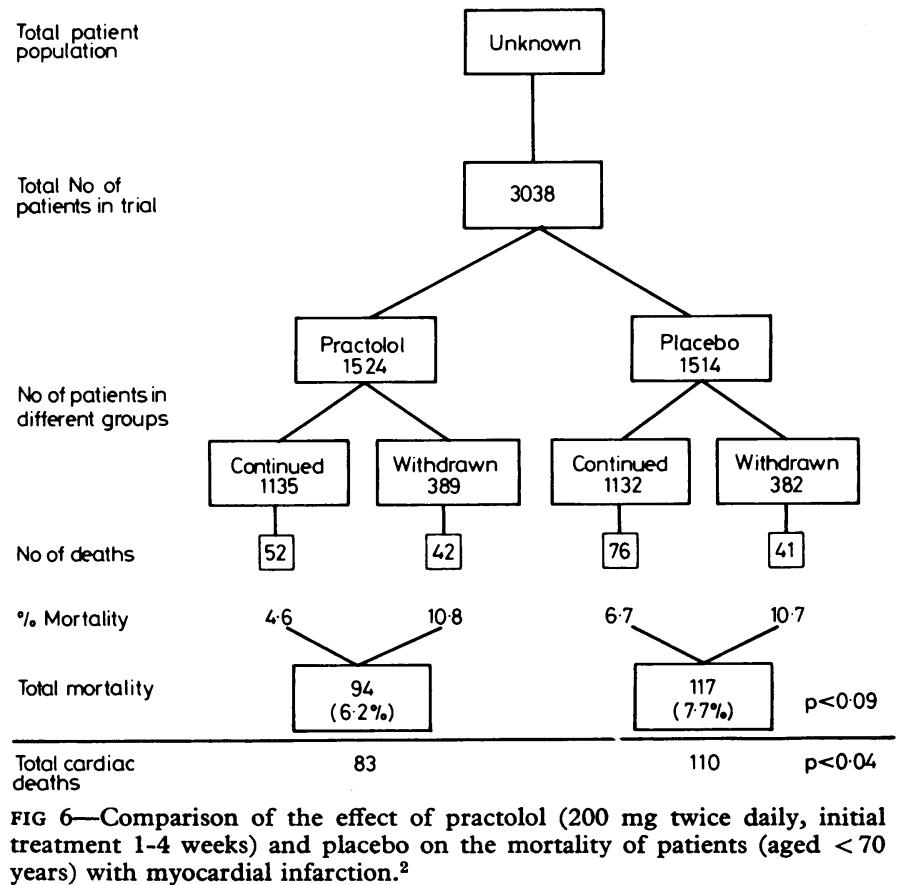
years) with myocardial infarction. ${ }^{2}$

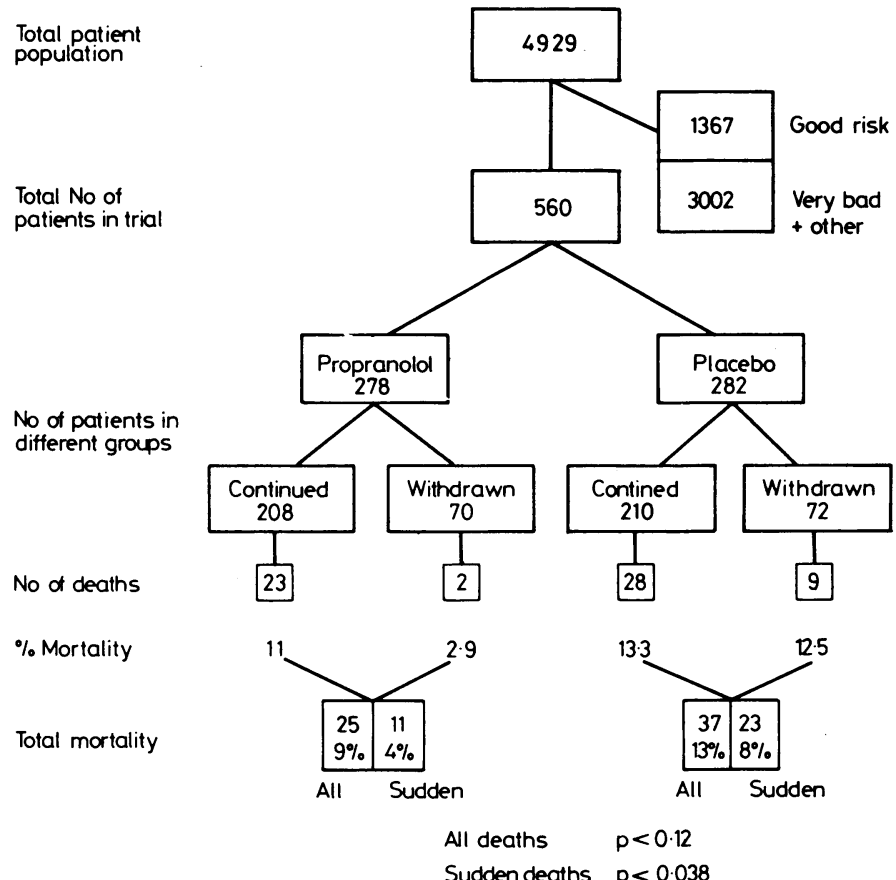

FIG 7-Comparison of the effect of propranolol ( $160 \mathrm{mg}$ daily) and placebo on total mortality and sudden death in patients with myocardial infarction ("high risk," 4-6 days after myocardial infarction up to one year).10

longed. If the trial results were precise (and the $95 \%$ confidence intervals show that this was not so) then 100 patients would have to be treated with timolol for 17 months to benefit six patients, and 100 patients would need treatment with propranolol for two years to prolong the life of two patients. It is therefore clearly desirable to identify if possible those patients likely to benefit from treatment and those who do not.

\section{Analysis of subgroups in clinical trials}

The only acceptable way to find out whether some particular subgroup of patients benefits from a particular treatment is to design a trial specifically for the purpose. Retrospective analysis of subgroups 
within a trial (data dredging) will not give valid answers, for if enough subgroups are formed retrospectively a difference between active and placebo treatment is likely to occur by chance. Such an analysis of the practolol trial ${ }^{2}$ suggested that the major benefit from active treatment was found in patients with anterior infarction patterns on the electrocardiogram, but when a later study with propranolol was designed specifically to investigate such patients no benefit from treatment was found ${ }^{12}$ (table I). Because of the withdrawal of practolol it is impossible to test the findings from the original study directly, but no selective benefit for patients with anterior infarctions has been found in any other trial, and it seems likely that this particular result from the practolol trial was spurious.

TABLE I-Effect of beta-blockers on mortality rate in patients with anterior or inferior myocardial infarct patterns by electrocardiography

\begin{tabular}{lccccc}
\hline & \multicolumn{4}{c}{$\%$ Mortality from: } \\
\cline { 2 - 3 } \cline { 5 - 6 } \cline { 5 - 6 } & \multicolumn{2}{c}{ Anterior infarction } & & \multicolumn{2}{c}{ Inferior infarction } \\
\cline { 2 - 3 } \cline { 5 - 6 } & Placebo & Beta-blockers & & Placebo & Beta-blockers \\
\hline Practolol & $6 \cdot 6$ & $3 \cdot 0^{*}$ & & $3 \cdot 7$ & $3 \cdot 3$ \\
Propranolol & $7 \cdot 4$ & 7.9 & & & \\
\hline
\end{tabular}

${ }^{*} \mathrm{p}<0.02$.

Separation of the trial population into groups (for example, by sex, age, weight, and severity of illness) before allocation to active or placebo treatment is called "prerandomisation stratification" and is a useful technique to ensure that the treatment groups are well matched. It is not valid to use the technique, however, to conduct multiple "trials within trials," for if enough subgroups are formed an apparently significant difference between active and placebo groups is likely to occur by chance. This probably happened in one of the trials of alprenolol, ${ }^{13}$ which seemed to show benefit from active treatment in a subgroup of younger patients and harm in a subgroup of older patients, for in no other trial has such an age effect been shown. Indeed, in the study of metoprolol ${ }^{7}$ it was the older patients who seemed to obtain most benefit from treatment (table II).

IABLE II-Effect of beta-blockers on mortality rate of patients of different ages after myocardial infarction

\begin{tabular}{lccccc}
\hline & \multicolumn{4}{c}{$\%$ Mortality } \\
\cline { 2 - 3 } \cline { 6 - 7 } & \multicolumn{2}{c}{$<65$ years } & & \multicolumn{2}{c}{$\geqslant 65$ years } \\
\cline { 2 - 3 } \cline { 5 - 6 } & Placebo & Beta-blockers & & Placebo & Beta-blockers \\
\hline Alprenolol & $20 \cdot 4$ & $9 \cdot 3^{*}$ & & 35 & 49 \\
Metoprolol & $5 \cdot 7$ & $4 \cdot 5$ & & $14 \cdot 3$ & $8 \cdot 1 \dagger$ \\
\hline
\end{tabular}

$* \mathrm{p}<0.01 . \quad+\mathrm{p}<0.05$.

TABLE III-Percentage reduction in mortality in patients with different risks of death in the Norwegian timolol trial and in the BHAT propranolol trial

\begin{tabular}{lcc}
\hline & \multicolumn{2}{c}{$\%$ Reduction in mortality } \\
\cline { 2 - 3 } & Timolol & Propranolol \\
\hline Overall reduction & 36 & 26 \\
Reinfarction & 21 & 18 \\
High risk & 40 & 30 \\
Low risk & 53 & 27 \\
\hline
\end{tabular}

The study of subgroups formed before entry to a trial is valid only if the expected mortality of placebo-treated patients in that subgroup can be accurately predicted and the size of the subgroup is sufficiently large for the expected benefit from treatment to be shown with conventional levels of statistical significance. An attempt to form such subgroups of patients with myocardial infarctions of different severity was made in the Norwegian timolol trial. ${ }^{9}$ Separate randomisation was performed for patients who had had a previous infarction, those who were considered to be at high risk, and those who were expected to be at low risk. Life-table analysis of these subgroups ${ }^{14}$ gives the impression that the major benefit from treatment occurred in the groups with previous infarction and at high risk and that in the lowrisk group treatment might not be worth while. Treatment was actually more effective in low-risk patients, however, and when by retrospective analysis similar subgroups were formed in the BHAT study a very similar pattern emerged (table III). Thus, although in the low-risk patients the benefits from treatment might be outweighed by possible side effects, there can be little doubt that treatment reduces still further the mortality in low-risk patients, and an individual patient might still prefer to be treated.

\section{Conclusions}

There can now be no doubt that late treatment with a betablocker reduces mortality in patients who have had a myocardial infarction, but there is still doubt about the value of early treatment. Unfortunately "late" and "early" have still not been accurately defined. Even the data from the recent group of clinical trials are inadequate to identify a subgroup of patients who should be treated and another that can be left untreated, and there seems little logical alternative but to treat all survivors of a myocardial infarction with a beta-blocker.

At least one-third of patients who have survived a myocardial infarction will need a beta-blocker because of hypertension or angina. A reduction in mortality after infarction is probably a general property of beta-blockers, although we cannot be sure of the correct dose of most of these drugs. The evidence at present is strongest for the use of propranolol (probably $80 \mathrm{mg}$ twice daily) or timolol (10 $\mathrm{mg}$ twice daily). Most survivors of infarcts are ready to leave hospital within a week, and it is usually both necessary and desirable that they should do so. My present practice is to begin prophylactic treatment with propranolol or timolol at the patient's first outpatient attendance.

\section{References}

1 Snow PJD. Effect of propranolol in myocardial infarction. Lancet 1965; ii:551-3.

2 A Multicentre International Study. Improvement in prognosis of myocardial infarction by long-term beta-adrenoreceptor blockade using practolol. $\mathrm{Br}$ Med $\mathcal{F}$ 1975; iii :735-40.

${ }^{3}$ Roland JM, Wilcox RG, Banks DC, Edwards B, Fentem PH, Hampton JR. Effect of beta-blockers on arrhythmias during six weeks after suspected myocardial infarction. Br Med f 1979; ii :518-21.

4 Wilcox RG, Roland JM, Banks DC, Hampton JR, Mitchell JRA. Randomised trial comparing propranolol with atenolol in immediate treatment of suspected myocardial infarction. $\mathrm{Br} M e d \mathcal{f} 1980 ; \mathbf{2 8 0}: 885-8$.

5 Beta-blocker Heart Attack Trial Research Group. A randomised trial of propranolol in patients with acute myocardial infarction. I Mortality results. F $A M A$ 1982;247:1707-14.

- Baber NS, Lewis JA. Beta-blockers in the treatment of myocardial infarction. Br Med f 1980;281:59.

${ }^{7}$ Hjalmarson A, et al. Effect on mortality of metoprolol in acute myocardial infarction. Lancet 1981 ;ii :823-6.

${ }^{8}$ Hampton JR. Presentation and analysis of the results of clinical trials in cardiovascular disease. $\mathrm{Br} \mathrm{Med} \mathcal{F} 1981$;282:1371-3.

9 The Norwegian Multicentre Study Group. Timolol-induced reduction in mortality and reinfarction in patients surviving acute myocardial infarction. $N$ Engl f Med 1981;304:801-7.

10 Hansteen V, et al. One year's treatment with propranolol after myocardial infarction: preliminary report of Norwegian multicentre trial. $\mathrm{Br} \mathrm{Med} \mathcal{F}$ $1982 ; 284: 155-60$.

11 Wilhelmsson C, Vedin JA, Wilhelmsen L, Tibblin G, Werko L. Reduction of sudden deaths after myocardial infarction by treatment with alprenolol. Lancet 1974 ;ii:1157-63.

12 Baber NS, Wainwright Evans D, Howitt G, et al. Multicentre postinfarction trial of propranolol in 49 hospitals in the United Kingdom, Italy and Yugoslavia. Br Heart $\mathcal{F} 1980 ; 44: 96-100$.

${ }^{13}$ Andersen MP, Frederiksen J, Jurgensen HJ, et al. Effect of alprenolol on mortality among patients with definite or suspected acute myocardial infarction. Lancet $1979 ; \mathrm{ii}: 865-8$.

14 Lund-Johansen $P$. The Norwegian multicentre study on timolol after myocardial infarction. Part II. Effect in different risk groups, causes of death, heart arrest, re-infarctions, rehospitalizations and adverse experiences. Proceedings of the symposium on acute and long-term management of myocardial ischaemia, Oslo, 1981.

(Accepted 8 Fune 1982) 